07,11

\title{
Откольная прочность ударно-разогретого гафния и уравнения состояния его полиморфных модификаций
}

\author{
() А.М. Молодец, А.А. Голышев \\ Институт проблем химической фризики РАН, \\ Черноголовка, Россия \\ E-mail: molodets@icp.ac.ru
}

Поступила в Редакцию 2 апреля 2019 г.

В окончательной редакции 2 апреля 2019 г.

Принята к публикации 2 апреля 2019 г.

\begin{abstract}
Выполнено определение откольной прочности гафния, разогретого нагружающей ударной волной до тысячных температур и испытавшего превращения в более плотные полиморфные модификации. Для достижения этой цели решены следующие задачи. Измерены профили давления на границе образец-мягкая преграда в откольных экспериментах при плоском одномерном нагружении. Построены уравнения состояния трех полиморфных модификаций гафния в диапазоне давлений до $100 \mathrm{GPa}$. Рассчитаны термодинамические состояния гафния в условиях выполненных экспериментов, что в комплекте с результатами измерений профилей давления позволило определить откольную прочность гафния, разогретого в цикле ударное сжатие-разгрузка. Величина откольной прочности гафния составила -4(1) GPa при температуре 1680(380) K. Выполнено математическое моделирование откольных экспериментов в рамках одномерного гидрокода.
\end{abstract}

Ключевые слова: откол, гафний, полиморфизм, ударные волны, фазовая диаграмма.

DOI: 10.21883/FTT.2019.08.47976.442

\section{1. Введение}

Как хорошо известно, гафний вместе с другими переходными металлами IV группы Ti, Zr, обладая развитым полиморфизмом, является функциональным и конструкционным материалом ряда важных отраслей промышленности. Поэтому полиморфизм и физико-механические свойства гафния в том числе при ударно-волновых нагрузках представляют собой важную информацию для фундаментальных и прикладных задач металлофизики переходных металлов IV группы элементов.

Ранее в [1] исследованы прочностные свойства гафния при ударно-волновом нагружении вне области полиморфных переходов и отмечался дефицит экспериментальных сведений о прочностных свойствах гафния при высокоскоростном нагружении. Цель данной работы заключалась в определении откольной прочности гафния, испытавшего в фазе ударного сжатия сильный разогрев и превращения в более плотные полиморфные модификации.

\section{2. Образцы для исследования и схема ударно-волнового нагружения образцов}

Как и в [1] исследовался гафний марки ГФИ-1 с массовой концентрацией примесей $0.9 \% \mathrm{Zr}, 0.034 \% \mathrm{Fe}$, $0.022 \% \mathrm{O}, \quad 0.02 \% \mathrm{C}, \quad 0.016 \% \mathrm{Si}, \quad 0.008 \% \mathrm{~N}$. Образцы гафния представляли собой плоскопараллельные пластины. Большие плоские поверхности образцов шлифовались. Плотность образцов составила $\rho_{0}=13.280(5) \mathrm{g} / \mathrm{cm}^{3}$.
Продольная скорость звука при нулевом давлении равна $C_{l}=3.765(5) \mathrm{km} / \mathrm{s}$. Для объемной скорости звука $C_{b}$ использовалось значение $C_{b}=\left(K_{0} / \rho_{0}\right)^{1 / 2}=2.86 \mathrm{~km} / \mathrm{s}$, где $K_{0}=108.78 \mathrm{GPa}[2]-$ справочное значение адиабатического модуля объемного сжатия гафния при нормальных условиях. Используемые образцы имели примерно равноосную микроструктуру с размером зерна $50 \mu \mathrm{m}$. Местоположение и относительная интенсивность рефлексов дифрактограммы поликристаллических образцов в исходном состоянии соответствовали справочным данным для гексагональной плотноупакованной ГПУ структуры $\alpha$-фазы гафния $\alpha$-Hf.

На рис. 1,a представлена схема ударноволновых экспериментов по исследованию откольной прочности с использованием традиционной методики образец-,мягкая“ преграда (см., например, [3]). Здесь ударник 1, разогнанный продуктами взрыва до скорости $W_{0}$, coударялся с исследуемым образцом 2 и генерировал в нем плоскую ударную волну. Образец соприкасался с мягкой преградой 3. На границы раздела 2-3, ударная волна распадалась на встречную волну разгрузки, уходящую в образец, и волну сжатия, распространяющуюся в преграду. В эксперименте регистрировалось падение электрического напряжения $U$ на чувствительном элементе манганинового датчика 4 во время прохождения по нему волны сжатия. Электрическое напряжение пересчитывалось в электросопротивление датчика, а затем в профиль $P(t)$ (зависимость давления $P$, от времени $t$ ) с помощью калибровки манганинового датчика из [4].

Откольное разрушение образца возникало в области взаимодействия встречной волны разгрузки с проходящей волной разгрузки от тыльной стороны ударника. 


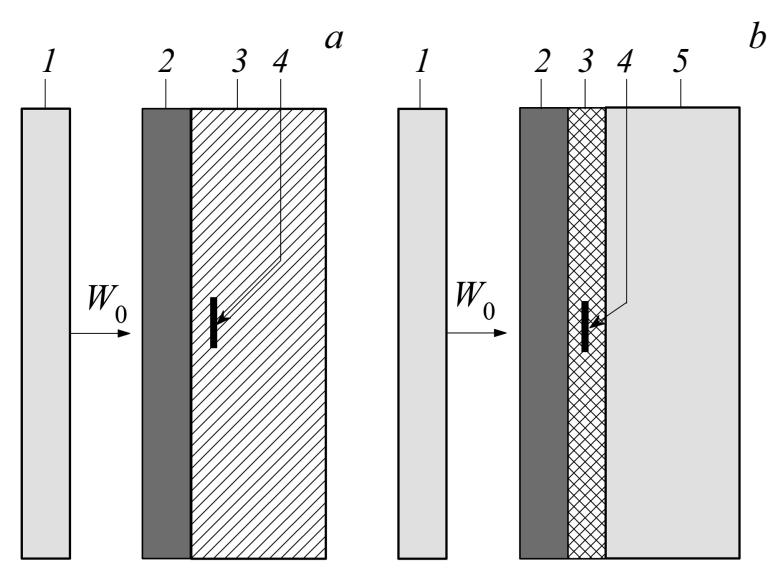

Рис. 1. Схема нагружения образцов гафния по регистрации профилей давления на границе образцов гафния с мягкой средой. a) 1 - стальной (нержавеющая сталь $12 \mathrm{X} 18 \mathrm{H} 10 \mathrm{~T})$ ударник толщиной $h_{0}=3.0(1) \mathrm{mm}$, разогнанный до скорости $W_{0}=3.2(1) \mathrm{km} / \mathrm{s} ; 2$ - образец ГФИ-1 толщиной $H_{0}=4.95(5) \mathrm{mm} ; 3$ - пластина плексигласа (РММА) толщиной 10.0(1) $\mathrm{mm} ; 4$ - манганиновый датчик толщиной $0.03 \mathrm{~mm}$ располагался на расстоянии $2 \mathrm{~mm}$ от поверхности образца; $b) 1,2$ - те же что и на рис. $1, a, 3-$ изолирующая прослойка из ТВС среды толщиной $0.4 \mathrm{~mm}, 4$ - манганиновый датчик толщиной $0.03 \mathrm{~mm}$, расположенный на расстоянии $0.2 \mathrm{~mm}$ от поверхности образца, 5 - алюминиевая пластина толщиной 14(1) $\mathrm{mm}$.

Информация об откольном разрушении образца отражалась на профиле $P(t)$ в форме откольного импульса, что позволяло определить откольную прочность образца.

На рис. $1, a$ „мягкой“ преградой служил плексиглас (PMМА). На рис. $1, b$ „мягкой“ преградой является слоистая преграда, состоящая из изолирующей прослойки 3, в виде двух тефлоновых пленок, склеенных вакуумной смазкой (ТВС [4] среда) и алюминиевой пластины 5. Использование тефлона позволило исключить паразитные поляризационные сигналы, имевшие место при использовании РММА. Использование алюминия позволило получить экспериментальные данные по откольному разрушению образца гафния в усложненных условиях слоистой преграды.

На рис. $2, a$ представлены профили $P(t)$ в экспериментах по схеме рис. $1, a$. На рис. $2, b$ представлены профили $P(t)$ в экспериментах по схеме рис. $1, b$.

\section{3. Обсуждение эксперимента}

Профили $P(t)$ на рис. $2, a$ содержат псевдопредвестник в области $t=0$. Этот предвестник обусловлен поляризационной наводкой от плексигласа, искажающий первичный электрический сигнал $U$ манганинового датчика. Использование в качестве материала преграды неполярного материала (тефлона) приводит, как это видно на pис. $2, b$, к исчезновению этого псевдопредвестника.

Возвращаясь к рис. $2, a$, отметим, что профили $P(t)$ на этом рисунке содержат характерные значения $P_{1}$ и $P_{2}$, которые служат базовыми величинами при определении откольной прочности $\sigma_{s}$ образца. Так, величина $\sigma_{s}$ может быть определена графически (см., например, [3]), если известны $P_{1}, P_{2}$, ударные адиабаты образца и преграды, а также их изэнтропы разгрузки в координатах давление $(P)$-массовая скорость $(u)$. Для целей настоящей работы необходимые ударные адиабаты в виде эмпирических зависимостей скорости ударной волны $D$ от массовой скорости

$$
D=C_{0}+s u
$$

были взяты из таблицы GMX-6, представленной на стр. 377 в [3]. Коэффициенты $C_{0}, s$, и начальная плотность $\rho_{0}$ рассматриваемых материалов приведены в табл. 1. Ударные адиабаты используемых материалов в координатах $P-u$ рассчитывались как

$$
P=\rho_{0} D u \text {. }
$$

Изэнтропы материалов в этих же координатах считались совпадающими с ударными адиабатами. Рассчитанные
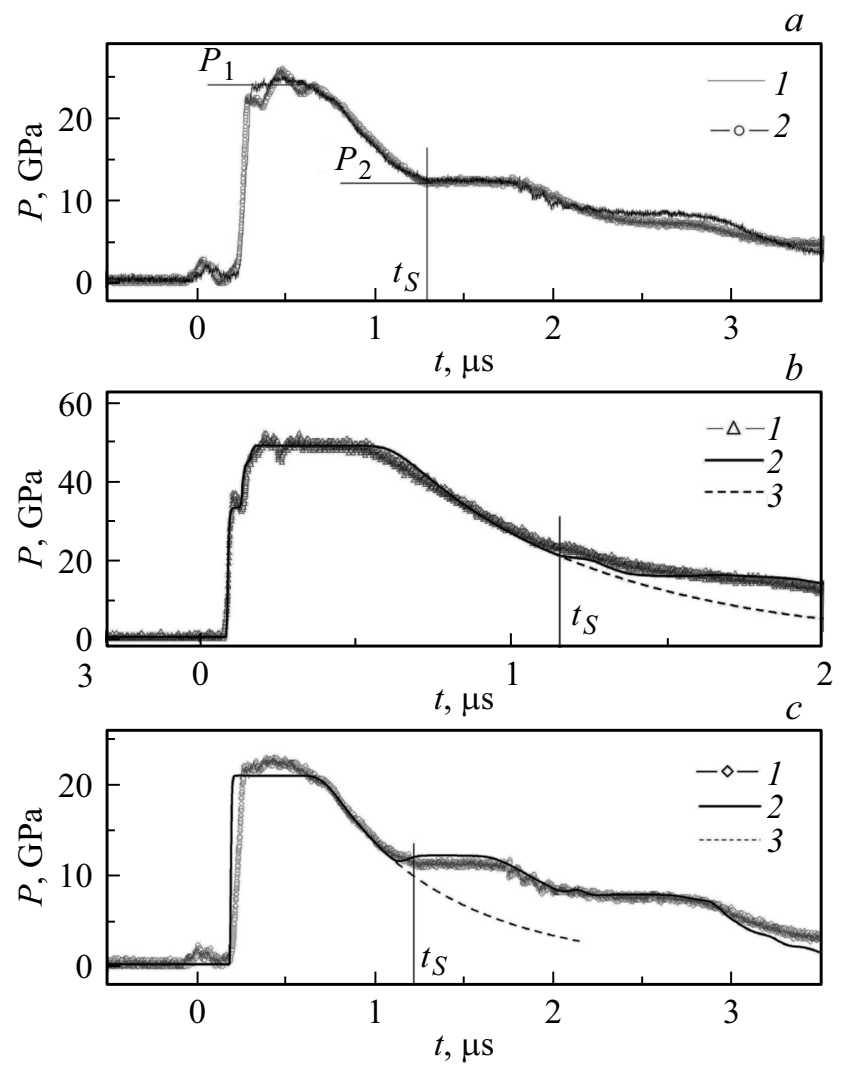

Рис. 2. Профили давления $P(t)$ в экспериментах по схеме рис. 1. $a-$ эксперименты по схеме рис. 1, $a: 1,2$ профили $P(t)$ от двух идентичных манганиновых датчиков $P_{1}-$ максимальное значение $P, P_{2}-$ значение $P$ в момент $t_{S}$ прихода откольного импульса на датчик; $b$ - эксперимент по схеме рис. $1, b$ : 1 - экспериментальный профиль $P(t), 2-$ модельный профиль при $\sigma_{s}=-4 \mathrm{GPa}, 3-$ „безоткольный“ модельный профиль, $c$ - эксперимент по схеме рис. $1, a, 1$ - экспериментальный профиль $P(t), 2$ - модельный профиль при $\sigma_{s}=-4 \mathrm{GPa}, 3-$ „безоткольный“ модельный профиль. 
Таблица 1. Ударные адиабаты материалов из [3]

\begin{tabular}{l|r|c|c|l}
\hline \multicolumn{1}{|c|}{ Материал } & $\rho_{0}, \mathrm{~g} / \mathrm{cm}^{3}$ & $C_{0}, \mathrm{~km} / \mathrm{s}$ & \multicolumn{1}{c|}{$s$} & \multicolumn{1}{c}{ Comments } \\
\hline Гафний-1 & 12.885 & 2.954 & 1.121 & Ниже $u=0.81 \mathrm{~km} / \mathrm{s}$ \\
Гафний-2 & 12.885 & 2.453 & 1.353 & Выше перехода \\
Нерж. сталь & 7.896 & 4.569 & 1.490 & \\
Плексиглас & 1.186 & 2.598 & 1.516 & Ниже $u=2.9 \mathrm{~km} / \mathrm{s}$
\end{tabular}

с использованием формул (1), (2) и данных табл. 1 ударные адиабаты и изэнтропы разгрузки приведены на рис. 3 в виде графиков 1,2, 5, 6 .

Рассмотрим с учетом $(1),(2)$ и данных рис. 2, a графическое определение откольной прочности на диаграмме рис. 3. Здесь пересечение 1 и 2 определяет состояние 3 ударно-сжатого гафния в ударной волне с амплитудой $P_{\text {sh }}=91(2) \mathrm{GPa}$. После распада этой ударной волны на границе образец-преграда в гафнии и плексигласе реализуется состояние 4 с давлением $P_{1}$, которое регистрируется манганиновым датчиком. В дальнейшем давление в плексигласе уменьшается вдоль изэнтропы 5 плексигласа до величины $P_{2}$, соответствующей моменту прихода откольного импульса (значение $P_{2}$ на рис. 3 приведено с учетом гистерезиса манганинового датчика в разгрузке). Через состояния плексигласа при $P_{1}$ и $P_{2}$ проведены изэнтропы разгрузки гафния 6 , пересечение которых определяет значение откольной прочности гафния $\sigma_{s}=-4(1) \mathrm{GPa}$.

Таким образом, данные рис. 2, $a$ и рис. 3 позволяют заключить, что откольная прочность гафния, предвари-

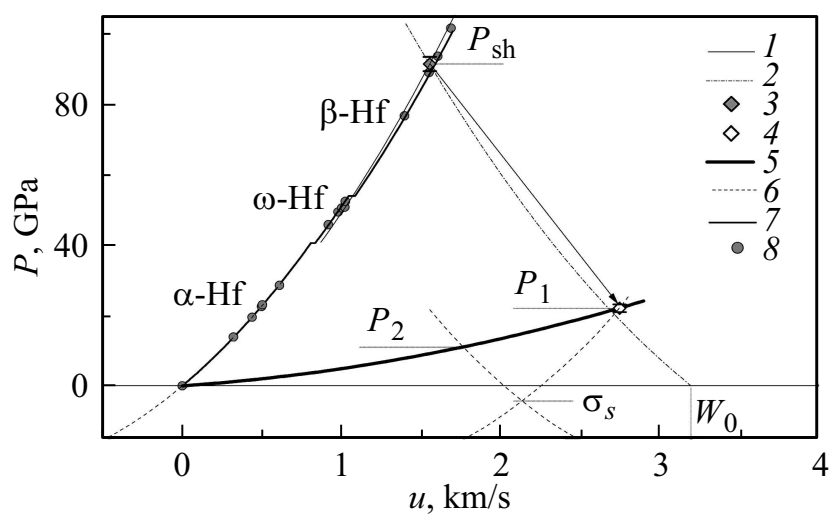

Рис. 3. Диаграмма давление $(P)$-массовая скорость $(u)$ ударного сжатия и разгрузки гафния при отколе. 1 - ударная адиабата Hafnium-2; 2 - ударная адиабата торможения ударника из нержавеющей стали с начальной скоростью $W_{0}=3.2(1) \mathrm{km} / \mathrm{s}$; 3 - состояние ударно-сжатого гафния при $P_{\mathrm{sh}}=91(2) \mathrm{GPa}$; 4 - состояние гафния разгруженного на плексиглас (стрелкой схематически указано изменение состояния гафния после распада ударной волны на границе с плексигласом); 5 ударная адиабата плексигласа, совпадающая с его изэнтропой разгрузки; 6 - ударная адиабата Hafnium-1 и его изэнтропы разгрузки; 7 - полуэмпирический расчет ударной адиабаты гафния, проходящей через области существования полиморфных модификаций $\alpha$-Hf, $\omega$-Hf, и $\beta$-Hf; $8-$ экспериментальные точки ударной адиабаты гафния из[5]. тельно сжатого ударной волной амплитудой 91(2) GPa, составляет величину $\sigma_{s}=-4(1) \mathrm{GPa}$.

Как хорошо известно, ударно-волновое сжатие вещества сопровождается его разогревом, увеличивающимся с увеличением амплитуды ударной волны. Грубые оценки температуры ударно-волнового разогрева гафния в проведенных экспериментах дают значения температур на уровне $2000 \mathrm{~K}$. Для уточнения этого значение, а также для оценки температуры гафния в разгрузке, где развивается откольное разрушение, были построены уравнения состояния полиморфных модификаций гафния, а затем рассчитана температура вдоль ударной адиабаты и вдоль последующей изэнтропы разгрузки до области растяжения. Ниже приведены техника построения уравнений состояния и соответствующие расчеты.

\section{4. Уравнения состояния, фазовая диаграмма гафния и моделирование эксперимента по откольному разрушению}

Как упоминалось выше гафний при нормальных условиях имеет гексагональную плотноупакованную ГПУ(hср) структуру ( $\alpha$-Hf фаза). При повышении температуры и атмосферном давлении происходит мартенситное превращение $\alpha$-Hf в $\beta$-Hf фазу с объемно центрированной кубической ОЦК(bcc) упаковкой атомов. Температура этого преврашения составляет 2014 К. При увеличении давления вдоль комнатной изотермы гафний претерпевает последовательность полиморфных переходов $\alpha$-Hf $\rightarrow \omega$-Hf $\rightarrow \beta$-Hf, то есть сначала в $\omega$-Hf фазу с гранецентрированной кубической ГЦК(fcc) упаковкой и затем в $\beta$-Нf фазу.

Расчеты фазовой диаграммы гафния до мегабарных давлений и тысячеградусных температур, где происходят $\alpha$-Hf $\leftrightarrow \omega$-Hf $\leftrightarrow \beta$-Hf полиморфные превращения этого металла предпринимались неоднократно (см. [6-8] и ссылки в них). Однако следует отметить, что фазовые диаграммы гафния различных авторов согласуются между собой лишь качественно. В частности, местоположения тройной точки и линий равновесия $\alpha$-Hf $\leftrightarrow \beta$-Hf в этих трех работах существенно различаются. Кроме этого, в $[7,8]$ не вовлечены в рассмотрение ударно-волновые экспериментальные данные $[9,10]$, которые важны для анализа экспериментальных результатов представляемой статьи. Поэтому в данной работе предпринято построение уравнений состояния и фазовой диаграммы гафния, которые дополняют имеющиеся прототипы, а также позволяют провести математическое моделирование откольных экспериментов в рамках ранее разработанного гидрокода

\section{1. Уравнения состояния полиморфных модификаций гафния}

Индивидуальные уравнения состояния для каждой полиморфной модификации гафния рассчитывались на 
основе полуэмпирического выражения для свободной энергии всесторонне сжатого изотропного твердого тела $\Phi=\Phi(V, T)$, представляющего сумму фононной $F=F(V, T)$ и электронной $F_{e}=F_{e}(V, T)$ составляющих

$$
\Phi=F(V, T)+F_{e}(V, T) .
$$

Фононная составляющая, базирующаяся на модели эйнштейновских осцилляторов, имеет вид

$$
F=3 R\left[\frac{\Theta}{2}+T \ln \left(1-\exp \left(-\frac{\Theta}{T}\right)\right)\right]+E_{x},
$$

где $R$ - удельная газовая постоянная, $V$ - удельный объем материала, $T$ - его температура, $\Theta=\Theta(V)-$ объемная зависимость характеристической температуры, определяемая согласно $[11,12]$ как

$$
\begin{gathered}
\Theta=\Theta_{0}\left(\frac{v_{0}-V}{v_{0}-V_{0}}\right)^{2}\left(\frac{V_{0}}{V}\right)^{2 / 3}, \\
v_{0}=V_{0}\left(1+\frac{2}{\gamma_{0}-2 / 3}\right) .
\end{gathered}
$$

В (1) - (4) $\Theta_{0}=\Theta\left(V_{0}\right)$ - характеристическая температура, $\gamma_{0}=\gamma\left(V_{0}\right)$ - термодинамический коэффициент Грюнайзена, $V_{0}$ - удельный объем в начальных условиях. Под начальными условиями $\left(T_{0}, P_{0}\right)$ везде ниже подразумевается состояние с комнатной температурой $T_{0}$ и атмосферным давлением $P_{0}$.

Объемная зависимость потенциальной энергии $E_{x}=E_{x}(V)$ также взята из $[11,12]$ в виде

$$
\begin{gathered}
E_{x}=-v_{x}\left(C_{1} H_{x}+C_{2} x\right)+C_{3}+E_{m}, \\
H_{x}=9\left(\frac{1}{10} x^{-\frac{2}{3}}+2 x^{\frac{1}{3}}+\frac{3}{2} x^{\frac{4}{3}}-\frac{1}{7} x^{\frac{7}{3}}+\frac{1}{70} x^{\frac{10}{3}}\right), \\
x=\frac{V}{v_{x}},
\end{gathered}
$$

В (7)-(9) $v_{x}$ - подгоночный параметр, первое приближение которого есть $v_{0}$. Параметры $C_{1}, C_{2}, C_{3}-$ константы, выражающиеся через справочные характеристики материала (плотность, модуль объемного сжатия, характеристическая температура, коэффициент Грюнайзена при начальных условиях) и подгоночный параметр $v_{x}$. Постоянное слагаемое $E_{m}$ задает уровень отсчета энергии для рассматриваемых фаз высокого давления.

Электронная составляющая $F_{e}$ записывалась согласно литературным данным [13]

$$
F_{e}=-\frac{1}{2} \beta_{0} T^{2}\left(\frac{V}{V_{0}}\right)^{1 / 2},
$$

где $V_{0}-$ начальный удельный объем, $\beta_{0}-$ коэффициент электронной теплоемкости. Уравнения состояния находились с помощью частных производных (3) согласно общим термодинамическим соотношениям. Калорическое уравнение состояния (зависимость внутренней энергии $E$ от объема и температуры) определяется

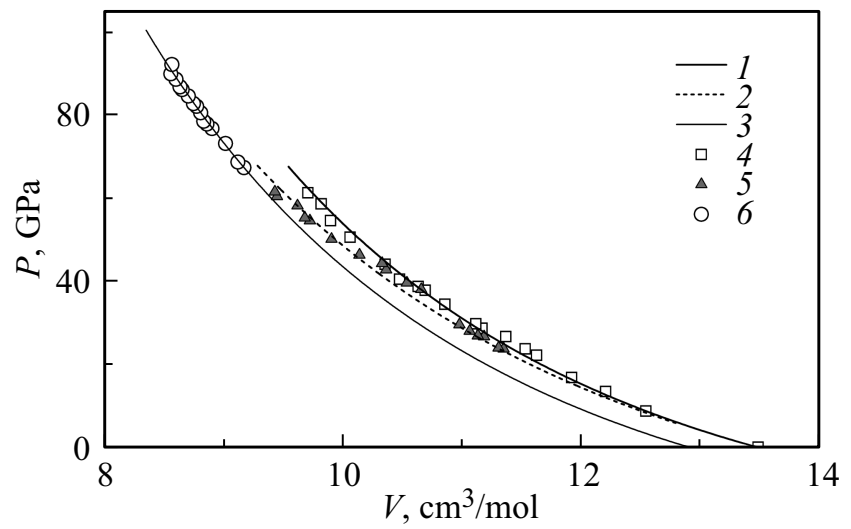

Рис. 4. Комнатные изотермы высокого давления (зависимость давления $P$ от объема $V$ при температуре $T_{0}=300 \mathrm{~K}$ ) полиморфных модификаций гафния. $1-3-$ расчет данной работы соответственно $\alpha$-Hf, $\omega$-Hf и $\beta$-Hf с помощью (12), 4-6 эксперимент соответственно для $\alpha$-Hf, $\omega$-Hf и $\beta$-Hf из [14].

частной производной свободной энергии по температуре $E=\Phi-T(\partial \Phi / \partial T)$. Теплоемкость при постоянном давлении $C_{p}$ рассчитывалась как

$$
C_{p}=-\left(\frac{\partial^{2} \Phi}{\partial T^{2}}+\frac{(\partial P / \partial T)^{2}}{(\partial T / \partial V)}\right) .
$$

Термическое уравнение состояния (зависимость давления $P$ от объема и температуры $T$ ) определяется частной производной свободной энергии по объему $P=P(V, T)=-\partial \Phi / \partial V$, которое после дифференцирования приобретает вид

$$
\begin{gathered}
P=C_{1} F(x)+C_{2}+3 R \frac{\gamma}{V} \Theta\left(\frac{1}{2}+\frac{1}{\exp (\Theta / T)-1}\right)+P_{e} \\
F(x)=3\left(-\frac{1}{5} x^{-\frac{5}{3}}+2 x^{-\frac{2}{3}}+6 x^{\frac{1}{3}}-x^{\frac{4}{3}}+\frac{1}{7} x^{\frac{7}{3}}\right), \\
\gamma=\frac{2}{3}+\frac{2 V}{v_{0}-V}, \\
P_{e}=\frac{\beta_{0}}{V_{0}}\left(\frac{T}{2}\right)^{2}\left(\frac{V_{0}}{V}\right)^{1 / 2} .
\end{gathered}
$$

Величины подгоночного параметра $v_{x}$ для каждой модификации гафния находились из условия наилучшего совпадения расчетной комнатной изотермы с экспериментальными данными из [14]. При этом расчетные изотермы рассчитывались с помощью уравнения состояния (10), в котором значение $T$ считалось постоянным и равным значению комнатной температуры $T_{0}$. Найденные величины $v_{x}$ вместе с формульной молярной массой $M_{f}, \Theta_{0}, V_{0}, v_{0}$ и $C_{1}, C_{2}, C_{3}$ для полиморфных модификаций гафния представлены в табл. 2, 3 .

Рис. 4 демонстрирует удовлетворительное согласие между расчетными и экспериментальными [14] изотермами $\alpha$-Hf, $\omega$-Hf и $\beta$-Hf фаз гафния. Видно, что расчет совпадает с экспериментом в пределах разброса экспериментальных данных. 
Таблица 2. Параметры для тепловых составляющих свободной энергии (3) $\alpha$-Hf, $\omega$-Hf и $\beta$-Hf фаз гафния (формула Hf), плексигласа (РМMA, формула $\mathrm{C}_{5} \mathrm{H}_{8} \mathrm{O}_{2}$ ) и тефлона (формула $\mathrm{CF}_{2}$ ) при нормальных условиях

\begin{tabular}{l|c|c|c|c|c}
\hline \multicolumn{1}{|c|}{ Фаза } & $\begin{array}{c}M_{f}, \\
\mathrm{~g} / \mathrm{mol}\end{array}$ & $\begin{array}{c}V_{0}, \\
\mathrm{~cm}^{3} / \mathrm{mol}\end{array}$ & $\begin{array}{c}\nu_{0}, \\
\mathrm{~cm}^{3} / \mathrm{mol}\end{array}$ & $\begin{array}{c}\Theta_{0}, \\
\mathrm{~K}\end{array}$ & $\begin{array}{c}\beta_{0}, \\
\mathrm{~mJ} / \mathrm{mol} \cdot \mathrm{K}\end{array}$ \\
\hline$\alpha$-Hf & 178.490 & 13.470 & 89.5015 & 147.3 & 2.64 \\
$\omega$-Hf & 178.490 & 13.460 & 124.0901 & 138.0 & 2.64 \\
$\beta$-Hf & 178.490 & 12.900 & 37.7724 & 101.3 & 6.019 \\
РММА & 100.117 & 84.416 & 492.879 & 630.0 & 0 \\
Тефлон & 50.007 & 22.73 & 89.259 & 142.5 & 0
\end{tabular}

\section{2. Фазовая диаграмма гафния}

Как хорошо известно, знание свободной энергии фаз как функции своих переменных позволяет рассчитать линии равновесия между этими фазами. Техника расчета фазовой диаграммы с используемым в данной работе функциональным видом (3) свободной энергии была проиллюстрирована в [12]. С использованием техники [12] рассчитана фазовая диаграмма гафния в области высоких давлений и температур, содержащая три линии равновесия между фазами $\alpha$-Hf, $\omega$-Hf, и $\beta$-Hf (см. рис. 5).

В выполненных расчетах основным состоянием считалась $\alpha$-фаза с величиной $E_{m}=0$ из (5). Коэффициент электронной теплоемкости $\beta_{0}$ из (10) для $\alpha$-фазы считался известным и был взят из справочной литературы $\beta_{0}=2.64 \mathrm{~mJ} / \mathrm{mol} \cdot \mathrm{K}$. Значения $E_{m}$ и $\beta_{0}$ для $\omega$-фазы и $\beta$-фазы рассматривались как подгоночные коэффициенты, которые определялись исходя из требования равенства химических потенциалов на известных точках линий равновесия фаз. Для $\alpha$-Hf и $\beta$-Hf фаз такой точкой служило состояние при давлении 1 атм и температуре $2014 \mathrm{~K}$. Для $\alpha$-Hf, и $\omega$-Hf такой точкой служило состояние при давлении $44.5 \mathrm{GPa}[14]$ и температуре $300 \mathrm{~K}$. Значения $E_{m}$ и $\beta_{0}$ для $\omega$-Hf и $\beta$-Hf также приведены в табл. 2 и 3.

Определенные вышеописанным способом соответствующие линии равновесия фаз гафния показаны линиями 1 на рис. 5. Здесь же показан расчетная ударная адиабата гафния 2 , пересекающая линии равновесия фаз. Как видно, 2 проходит через точку 3 фазового перехода ударно-сжатого гафния из [9], и согласуется с оценками температуры и давления в точке излома ударной адиабаты гафния 4 из [10]. Кроме этого расчетная ударная

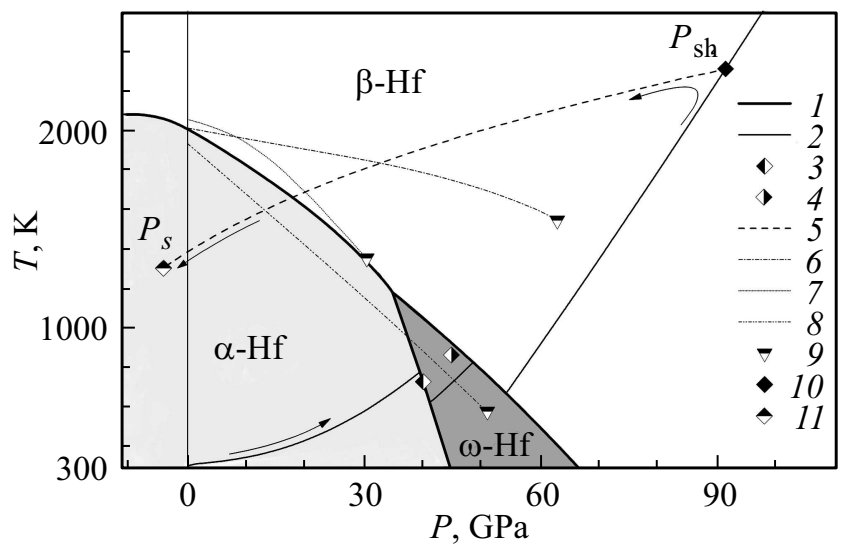

Рис. 5. Фазовая диаграмма гафния в координатах давление $P$-температура $T .1$ - линии равновесия $\alpha$-Hf, $\omega$-Hf, $\beta$-Hf фаз, рассчитанные в данной работе; 2 - расчетная ударная адиабата гафния, пересекающая линии равновесия фазовых превращений; 3 - давление и температура фазового превращения ударно-сжатого гафния из [9]; 4 - оценка температуры и давления в точке излома ударной адиабаты гафния из [10]; 5 - расчетная изэнтропа разгрузки $\beta$-Hf; $6,7,8-$ линии равновесия $\alpha$-Hf $\leftrightarrow \beta$-Hf соответственно из [6], [7], [8]; 9 - тройные точки из [6-8]; $10-$ состояние $\left(P_{\mathrm{sh}}, T_{\mathrm{sh}}\right)$ ударно-сжатого гафния в экспериментах данной работы; $11-$ состояние $\left(P_{s}, T_{s}\right)$ метастабильной фазы $\beta$-Нf в области растяжения; стрелками обозначен путь ударно-волнового сжатия и последующей разгрузки.

адиабата гафния в координатах давление $(P)$-массовая скорость $(u)$ (см. 7 на рис. 3) оптимально располагается среди экспериментальных точек [5] (см. 8 на рис. 3). Иными словами, можно сказать, что расчеты ударных адиабат всех фаз гафния согласуются качественно и количественно с экспериментом, что свидетельствует в пользу достоверности разработанного термодинамического описания гафния при давлениях ударного сжатия до $\sim 100 \mathrm{GPa}$. Расчеты в рамках этого описания дают значение температуры ударного сжатия гафния $T_{\mathrm{sh}}$ при давлении $P_{\mathrm{sh}}$ равное $T_{\mathrm{sh}}=2300 \mathrm{~K}$.

Обсудим значение температуры гафния в разгрузке, когда развивается откольное разрушение в экспериментах по схеме рис. 1. На рис. 5 графиком 5 представлена расчетная изэнтропа разгрузки ударно-сжатой фазы $\beta$-Hf, выходящая из точки $\left(T_{\mathrm{sh}}, P_{\mathrm{sh}}\right)$. Как видно 5 достигает линию равновесия $\alpha \leftrightarrow \beta$, минуя область существования

Таблица 3. Параметры для потенциальной энергии (7) $\alpha$-Hf, $\omega$-Hf и $\beta$-Hf (формула $\mathrm{Hf})$, плексигласа $\left(\mathrm{PMMA}\right.$, формула $\left.\mathrm{C}_{5} \mathrm{H}_{8} \mathrm{O}_{2}\right)$ и тефлона (формула $\mathrm{CF}_{2}$ )

\begin{tabular}{l|c|c|c|c|c|c}
\hline Фаза & $v_{x}, \mathrm{~cm}^{3} / \mathrm{mol}$ & $C_{1}, \mathrm{GPa}$ & $C_{2}, \mathrm{GPa}$ & $C_{3}, \mathrm{~kJ} / \mathrm{g}$ & $E_{m}, \mathrm{~kJ} / \mathrm{g}$ & $P_{\text {range }}, \mathrm{GPa}$ \\
\hline$\alpha$-Hf & 43.1040 & -68.9555 & 1410.7346 & -175.7854 & 0.0 & $-10-60$ \\
$\omega$-Hf & 56.5320 & -28.5181 & 563.7952 & -97.0094 & 0.0277 & $-10-60$ \\
$\beta$-Hf & 43.8600 & -58.2587 & 1185.2920 & -151.6091 & 0.153 & $-10-90$ \\
PMMA & 141.818 & -76.3468 & 1587.7589 & -1128.1953 & 0 & $1-25$ \\
Teflon & 28.277 & -703.9228 & 14660.2894 & -4145.8364 & 0 & $3-40$
\end{tabular}


фазы $\omega$-Hf. Отметим, что этот вывод не изменяется, если опираться на фазовые диаграммы гафния других авторов - 6 из [6], 7 из [7] и 8 из [8]. Действительно, изэнтропа 5 во всех случаях пресекает только линию равновесия $\alpha \leftrightarrow \beta$.

Заметим далее, что если для ударного сжатия эксперименты [9] свидетельствуют о фазовом переходе гафния, то для разгрузки такие данные отсутствуют. Поэтому, вообще говоря, после пересечения изэнтропы 5 с линией равновесия $\alpha \leftrightarrow \beta$ возможны два сценария. По первому сценарию обратный переход $\beta$-Hf $\rightarrow \alpha$-Hf не происходит. В этом случае откольное разрушение развивается в метастабильной фазе $\beta$-Hf при температуре, обозначенной точкой 11 , которая составляет $T_{s}=1300 \mathrm{~K}$. Если же $\beta$-Hf фаза превращается в разгрузке в исходную $\alpha$-Hf фазу с выделением тепла, то температура разрушающегося металла будет больше. Увеличение температуры можно оценить воспользовавшись соотношением $\Delta T=E_{m} / C_{p}$, где согласно табл. 3 величина $E_{m}=0.153 \mathrm{~kJ} / \mathrm{g}$, теплоемкость $C_{p}$ согласно формуле $(11)$ в точке $\left(T_{s}, P_{s}\right)$ равна $C_{p}=35.8 \mathrm{~J} / \mathrm{mK}$. Эти значения дают $\Delta T=760 \mathrm{~K}$. Таким образом, объединяя оценки $\Delta T$ и $T_{s}$, можно сказать, что в проведенных экспериментах температура гафния при его откольном разрушении составляла $T_{s}=1680(380) \mathrm{K}$.

В заключение отметим, что изэнтропа 5 на рис. 5 пересекает линию равновесия $\alpha \leftrightarrow \beta$ при давлении $\sim 20 \mathrm{GPa}$. Этот результат послужил обоснованием для того, чтобы при определении откольной прочности в графических построениях на рис. 3 использовать изэнтропу разгрузки фазы $\alpha$-Hf.

\section{3. Математическое моделирование ударно-волновых экспериментов}

В качестве иллюстрации комплексного использования полученных результатов применительно к прикладной задаче прогнозирования откольного разрушения гафния было выполнено математическое моделирование выполненных экспериментов. Моделирование осуществлялось в рамках одномерного гидрокода [15], опирающегося на уравнения состояния материалов ударника, образца и слоистой мягкой преграды в схеме рис. 1.

Уравнение состояния нержавеющей стали взято из [16]. Уравнения состояния плексигласа и тефлона (см. табл. 2,3) были специально разработаны для диапазона давлений ниже $26 \mathrm{GPa}$ для плексигласа и ниже $40 \mathrm{GPa}$ для тефлона. При этом величины подгоночного параметpa $v_{x}$ для плексигласа и тефлона находились из условия наилучшего совпадения расчетной ударной адиабаты с экспериментальными данными из [17]. Уравнение состояния алюминия взято из [18]. В качестве уравнения состояния образца использовано уравнение состояния $\alpha$-Hf, приведенное в предыдущем разделе.

Откольное разрушение гафния моделировалось в рамках модели мгновенного откола. Считалось, что в лагранжевой частице образца мгновенно образуются две свободные поверхности, как только давление в этой частице достигнет значения $\sigma_{s}=-4 \mathrm{GPa}$. Было проведено также моделирование „безоткольных“ экспериментов, соответствующих бесконечно большой (по абсолютной величине) откольной прочности. Результаты моделирования представлены на рис. $2, b$ и рис. $2, c$.

Как видно, в случае моделирования экспериментов с простой преградой в постановке рис. 1, $a$, модельный профиль 2 на рис. 2, с удовлетворительно воспроизводит откольный импульс в момент $t_{S}$ и последующую его циркуляцию в откольной пластине в виде ступенчатой разгрузки. Видно также существенное различие „безоткольного“ профиля 3 и экспериментального профиля 1 после момента $t_{S}$.

Модельный профиль 2 на рис. $2, b$, соответствующий усложненной преграде по схеме рис. $1, b$, также практически совпадает с экспериментальным профилем 1 . Отметим, что в этом случае „безоткольный“ профиль 3 незначительно отличается от профиля 2, содержащего откольный импульс. Этот результат позволяет идентифицировать малозаметную особенность на экспериментальном профиле 1 в области $t_{S}$ как откольное разрушение образца в усложненной постановке рис. 2, $b$. Таким образом, рассматриваемая модель, во-первых, обладает прогностическими возможностями и, во-вторых, позволяет квалифицировать экспериментальные профили в постановке эксперимента рис. $1, b$ как экспериментальные данные по откольному разрушению образца гафния в усложненных условиях слоистой преграды.

\section{5. Заключение}

Проведены эксперименты по измерению откольной прочности образцов гафния ГФИ-1 в плоской одномерной постановке. Амплитуда нагружающей ударной волны в гафнии составила величину 91(2) GPa. Температура ударного сжатия образцов составила $2300 \mathrm{~K}$. Откольная прочность гафния, предварительно сжатого и разогретого в ударной волне, составила величину $\sigma_{s}=-4(1) \mathrm{GPa}$ при температуре $1680(380) \mathrm{K}$. Построены полуэмпирические уравнения состояния полиморфных модификаций гафния $\alpha$-Hf, $\omega$-Hf и $\beta$-Hf и линии равновесия этих модификаций в области давлений до $\sim 100 \mathrm{GPa}$ и температур до $\sim 2000 \mathrm{~K}$. С использованием полученных экспериментальных и расчетных результатов проведено прогностическое моделирование откола в выполненных экспериментах.

\section{Финансирование работы}

Работа выполнена при поддержке Госкорпорации „Росатом“ в рамках государственного контракта № H.4x.241.9Б.17.1013 от 20.02.2017 и Госзадания № 0089-2019-0001. с использованием оборудования Уникальной научной установки „Экспериментальный взрывной стенд“.

\section{Конфликт интересов}

Авторы заявляют, что у них нет конфликта интересов. 


\section{Список литературы}

[1] А.М. Молодец, А.С. Савиных, А.А. Голышев, Г.В. Гаркушин. Письма в ЖТФ 45, 2, 29 (2019).

[2] K.A. Gschneidner. Solid State Phys. 16, 275 (1964).

[3] D.E. Grady, M.E. Kipp. In: High-Pressure Shock Compression of Condensed Matter / Eds J.R. Asay and M. Shahinpoor. Springer-Verlag, N. Y. Berlin Heidelberg. (1992). P. 265.

[4] А.А. Голышев, А.М. Молодец. ФГВ 49, 2, 106 (2013).

[5] S.P. Marsh, Shock Hugoniot Data / Eds S.P. Marsh. Univ. California LASL Press, Berkeley (1980).

[6] S.A. Ostanin, V.Y. Trubitsin. Comput. Mater. Sci. 17, 174 (2000).

[7] Hrubiak R. Exploring thermal and mechanical properties of selected transition elements under extreme conditions: experiments at high pressures and high temperatures $[\mathrm{PhD}$ thesis]. Miami, Florida International University (2012).

[8] Cheng-Bin Zhang, Wei-Dong Li, Ping Zhang, Bao-Tian Wang. Comput. Mater. Sci. 157, 121 (2019).

[9] R. Mc Queen, S. Marsh, J. Taylor, J. Fritz, W. Carter. in High Velocity Impact Phenomena. High-Velocity Impact Phenomena / Ed. R. Kinslow. Academic Prss, N.Y. and London (1970). P. 293.

[10] А.А. Баканова, И.П. Дудоладов, Ю.Н. Сутулов. ФТТ 11, 1881 (1969).

[11] А.М. Молодец. ЖЭТФ 107, 3, 824 (1995).

[12] A.M. Molodets. High Press. Res. 30, 325 (2009).

[13] Л.В. Альтшулер, С.Б. Кормер, А.А. Баканова, Р.Ф. Трунин. ЖЭТФ 38, 3, 790 (1960).

[14] K. Pandey, J. Gyanchandani, M. Somayazulu, G.K. Dey, Surinder M. Sharma, S.K. Sikka. J. Appl. Phys. 115, 233513 (2014).

[15] В.В. Ким, А.А. Голышев, Д.В. Шахрай, А.М. Молодец. В сб.: Тр. XI Междунар. конф. „Забабахинские научные чтения“. Снежинск (2012); http://www.vniitf.ru/images/zst/2012/s6/6-24.pdf.

[16] А.М. Молодец. ФТТ 57, 10, 1992 (2015).

[17] W. Carter, S. Marsh. Hugoniot equation of state of polymers, Report № LA-13006-MS, Los Alamos National Laboratory, NM (1995).

[18] А.М. Молодец. ФТТ 56, 11, 2162 (2014).

Редактор Ю.Э. Китаев 